\title{
A FAMILY OF $*$-ALGEBRAS ALLOWING WICK ORDERING: FOCK REPRESENTATIONS AND UNIVERSAL ENVELOPING $C^{*}$-ALGEBRAS
}

\author{
PALLE E. T. JORGENSEN * \\ Department of Mathematics \\ The University of Iowa \\ Iowa City, Iowa 52242-1419 U.S.A. \\ DANIIL P. PROSKURIN ${ }^{\dagger}$ \\ Kyiv Taras Shevchenko University \\ Cybernetics Department \\ Volodymyrska, 64, Kyiv, 01033, Ukraine \\ YURII SAMOILENKO $\ddagger$ \\ Institute of Mathematics, National Academy of Sciences \\ Tereschenkivska, 3, Kyiv, 01601, Ukraine
}

\begin{abstract}
We consider an abstract Wick ordering as a family of relations on elements $a_{i}$ and define $*$-algebras by these relations. The relations are given by a fixed operator $T: \mathfrak{h} \otimes \mathfrak{h} \rightarrow \mathfrak{h} \otimes \mathfrak{h}$, where $\mathfrak{h}$ is oneparticle space, and they naturally define both a $*$-algebra and an innerproduct space $\mathcal{H}_{T},\langle\cdot, \cdot\rangle_{T}$. If $a_{i}^{*}$ denotes the adjoint, i.e., $\left\langle a_{i} \varphi, \psi\right\rangle_{T}=$ $\left\langle\varphi, a_{i}^{*} \psi\right\rangle_{T}$, then we identify when $\langle\cdot, \cdot\rangle_{T}$ is positive semidefinite (the positivity question!). In the case of deformations of the CCR-relations (the $q_{i j}$-CCR and the twisted CCR's), we work out the universal $C^{*}$ algebras $\mathfrak{A}$, and we prove that, in these cases, the Fock representations of the $\mathfrak{A}$ 's are faithful.
\end{abstract}

\section{Introduction}

In recent papers $[1,2,3,4,5,6]$, the applications of Lie superalgebras, quantum groups, $q$-algebras in mathematical physics have stimulated interest in

\footnotetext{
* (jorgen@math.uiowa.edu)

$\dagger$ (prosk@imath.kiev.ua)

¥ (yurii_sam@imath.kiev.ua)
} 
the $*$-algebras defined by generators and relations and their representations by Hilbert space operators. For example, the representations of various deformations of canonical commutation relations (CCR), in particular Fock representaion, were used to construct non-classical models of theoretical physics and probability, such as the free quon gas (see [7]), q-Gaussian processes (see [8]) etc.

The constructions are interesting from both physical and mathematical points of view. They give a canonical realisation of a given deformed relation like the Fock representation, or a realisation by differential operators. When the relations can be realised by bounded operators, it is useful to study the universal enveloping $C^{*}$-algebras for them and the stability of isomorphism classes of these $C^{*}$-algebras on parameters (see for example $[9,10]$ ). The stability question [10] refers to how the $C^{*}$-isomorphism classes depend on variations in the deformation variables; in some cases there are open regions in parameter space where the $C^{*}$-isomorphism class is constant.

In the present paper we give a review of some results concerning a wide class of deformed relations of the following form

$$
a_{i}^{*} a_{j}=\delta_{i j} 1+\sum_{k, l=1}^{d} T_{i j}^{k l} a_{l} a_{k}^{*}, i, j=1, \ldots, d,
$$

where $T_{i j}^{k l} \in \mathbb{C}$, such that $T_{i j}^{k l}=\bar{T}_{j i}^{l k}$. These relations generate a $*$-algebra allowing Wick ordering or Wick algebra (see $[4,11,12,13]$ ). The *algebra $\mathfrak{A}_{T}$ has a naturally defined Fock vacuum "state" or functional and there is a corresponding inner-product space $\mathcal{H}_{T},\langle\cdot, \cdot\rangle_{T}$, such that, in the associated GNS-representation, the identity $\left\langle a_{i} \varphi, \psi\right\rangle_{T}=\left\langle\varphi, a_{i}^{*} \psi\right\rangle_{T}$ holds. But the vacuum functional is generally not positive, and the operators in the representation not bounded, and therefore the Hermitian inner product $\langle\cdot, \cdot\rangle_{T}$ is then generally not positive semidefinite. The positivity question, and the faithfulness of the Fock representation, are the foci of this paper.

Note that (1) generalises some well-known types of deformed commutation relations, quantum groups, etc. (see $[1,3,5,6,8,12,14,15])$. The basic examples for us will be the $q_{i j}$-CCR introduced and studied by M. Bożejko and R. Speicher (see $[8,12]$ ), and the twisted canonical commutation relations (TCCR) constructed by W. Pusz and S.L. Woronowicz (see [6]). They were further studied in [16] where the traditional Cuntz algebra of [17] was considered as a base-point, corresponding to $q_{i j}=0$, and the variation of the $C^{*}$-isomorphism class was considered as a function of $q_{i j}$.

EXAMPlE 1. $q_{i j}$-CCR, $2 d$ generators:

$$
\begin{aligned}
& \mathbb{C}\left\langle a_{i}, a_{i}^{*}\right| a_{i}^{*} a_{j}=\delta_{i j} 1+q_{i j} a_{j} a_{i}^{*}, i, j=1, \ldots, d, \\
& \left.\quad q_{j i}=\bar{q}_{i j} \in \mathbb{C},\left|q_{i j}\right| \leq 1\right\rangle
\end{aligned}
$$


EXAMPLE 2. The Wick algebra for TCCR:

$$
\begin{aligned}
& a_{i}^{*} a_{i}=1+\mu^{2} a_{i} a_{i}^{*}-\left(1-\mu^{2}\right) \sum_{k<i} a_{k} a_{k}^{*}, i=1, \ldots, d \\
& a_{i}^{*} a_{j}=\mu a_{j} a_{i}^{*}, \quad i \neq j, \quad 0<\mu<1
\end{aligned}
$$

We present some sufficient conditions on the coefficients $\left\{T_{i j}^{k l}\right\}$ for the existence of the Fock representation, and we describe the structure of the Fock space. We also give conditions for the faithfulness of Fock representation and describe its kernel in the degenerated case (see Sec. 3).

Further we consider the universal $C^{*}$-algebras for the examples above. Specifically we show that the universal $C^{*}$-algebras for $q_{i j}$-CCR (TCCR) can be generated by isometries (partial isometries) satisfying a certain algebraic relation. The description of the $C^{*}$-isomorphism classes for different values of parameters is presented.

We also show that the Fock representations of $q_{i j}$-CCR for some values of parameters, and TCCR for any value of parameter, are faithful on the $C^{*}$-level, i.e., the Fock representations of the corresponding $C^{*}$-algebras are faithful (see Sec. 4).

The complete proofs of all results presented here can be found in [4, $10,11,18,19]$. For detailed information about $*$-representations of finitely generated $*$-algebras see [20].

\section{Basic definitions}

Firstly let us construct a canonical realisation of Wick algebra, i.e., the *-algebra on the relations (1), with coefficents $\left\{T_{i j}^{k l}\right\}$ : we denote it by $W(T)$. To do it consider a finite-dimensional Hilbert space $\mathcal{H}=\left\langle e_{1}, \ldots, e_{d}\right\rangle$. Construct the full tensor algebra over $\mathcal{H}, \mathcal{H}^{*}$, denoted by $\mathcal{T}\left(\mathcal{H}, \mathcal{H}^{*}\right)$. Then

$$
W(T) \cong \mathcal{T}\left(\mathcal{H}, \mathcal{H}^{*}\right) /\left\langle e_{i}^{*} \otimes e_{j}-\delta_{i j} 1-\sum T_{i j}^{k l} e_{i} \otimes e_{j}^{*}\right\rangle,
$$

dividing out by the two-sided ideal on the relations (1). Note that in this realisation the subalgebra of $W(T)$ generated by $\left\{a_{i}\right\}$ is identified with the $\mathcal{T}(\mathcal{H})$.

The following operators were presented in [11] as a useful tool for computation with Wick algebras and their Fock representations.

$$
\begin{gathered}
T: \mathcal{H} \otimes \mathcal{H} \mapsto \mathcal{H} \otimes \mathcal{H}, \quad T e_{k} \otimes e_{l}=\sum_{i, j} T_{i k}^{l j} e_{i} \otimes e_{j}, T=T^{*} \\
T_{i}: \mathcal{H}^{\otimes n} \mapsto \mathcal{H}^{\otimes n}, \quad T_{i}=\underbrace{1 \otimes \cdots \otimes 1}_{i-1} \otimes T \otimes \underbrace{1 \otimes \cdots \otimes 1}_{n-i-1}, \\
R_{n}: \mathcal{H}^{\otimes n} \mapsto \mathcal{H}^{\otimes n}, \quad R_{n}=1+T_{1}+T_{1} T_{2}+\cdots+T_{1} T_{2} \cdots T_{n-1}, \\
P_{n}: \mathcal{H}^{\otimes n} \mapsto \mathcal{H}^{\otimes n}, \quad P_{2}=R_{2}, P_{n+1}=\left(1 \otimes P_{n}\right) R_{n+1} .
\end{gathered}
$$


The sequences of operators $P_{0}=1_{\mathrm{vac}}, R_{1}=1+T, P_{1}=(1 \otimes 1)(1+T) \cong$ $1+T, R_{2}, \ldots, P_{n}$ are defined recursively. It is the sequence $P_{n}$ which enters into the positivity question. The other one is only intermediate. The Hermitian inner product $\langle\cdot, \cdot\rangle_{T}$ on $\mathcal{T}_{n}(\mathcal{H})$ is then

$$
\langle\phi, \psi\rangle_{\mathcal{T}_{n}(\mathcal{H})}:=\left\langle\varphi, P_{n} \psi\right\rangle_{\text {tensor }}
$$

where $\langle\cdot, \cdot\rangle_{\text {tensor }}$ is just the usual inner product on $\mathcal{T}_{n}(\mathcal{H})$ induced by $\langle\cdot, \cdot\rangle$ on $\mathcal{H}$. Hence, we need conditions on $T: \mathcal{H} \otimes \mathcal{H} \rightarrow \mathcal{H} \otimes \mathcal{H}$ which make the operators $P_{n}$ positive for all $n$. For example, in terms of these operators we can describe the procedure of Wick ordering, i.e., the commutation formula for fixed generator $a_{i}^{*}$ and any homogeneous polynomial in $a_{k}, k=1, \ldots, d$ (see [21]).

Proposition 1. Let $X \in \mathcal{H}^{\otimes n}$. Then

$$
e_{i}^{*} \otimes X=\mu\left(e_{i}^{*}\right) R_{n} X+\mu\left(e_{i}^{*}\right) \sum_{k=1}^{d} T_{1} T_{2} \cdots T_{n}\left(X \otimes e_{k}\right) e_{k}^{*},
$$

where $\mu\left(e_{i}^{*}\right): \mathcal{T}(\mathcal{H}) \mapsto \mathcal{T}(\mathcal{H})$ is defined as follows

$$
\mu\left(e_{i}^{*}\right) 1=0, \quad \mu\left(e_{i}^{*}\right) e_{i_{1}} \otimes \cdots \otimes e_{i_{n}}=\delta_{i i_{1}} e_{i_{2}} \otimes \cdots \otimes e_{i_{n}} .
$$

For our examples the operator $T$ have the following form:

EXAMPLE 3.

$$
T e_{i} \otimes e_{j}=q_{i j} e_{j} \otimes e_{i}, i, j=1, \ldots, d .
$$

EXAMPLE 4.

$$
\begin{aligned}
& T e_{i} \otimes e_{i}=\mu^{2} e_{i} \otimes e_{i} \\
& T e_{i} \otimes e_{j}=\mu e_{j} \otimes e_{i}, i<j \\
& T e_{i} \otimes e_{j}=-\left(1-\mu^{2}\right) e_{i} \otimes e_{j}+\mu e_{j} \otimes e_{i}, i>j .
\end{aligned}
$$

Note that for both examples, the operator $T$ satisfies a braid condition, i.e., on the $\mathcal{H}^{\otimes 3}$ we have

$$
T_{1} T_{2} T_{1}=T_{2} T_{1} T_{2}
$$

The operators presented above appear naturally in construction of Fock representation of $W(T)$. This notion is induced in the obvious way from the classical one for CCR, however, in general, the Fock space is not always symmetric (see [11]). 
Definition 1. The representation $\lambda_{0}$ acting on the space $\mathcal{T}(\mathcal{H})$ by formulas

$$
\begin{aligned}
\lambda_{0}\left(a_{i}\right) e_{i_{1}} \otimes \cdots \otimes e_{i_{n}} & =e_{i} \otimes e_{i_{1}} \otimes \cdots \otimes e_{i_{n}}, \quad n \in \mathbb{N} \cup\{0\} \\
\lambda_{0}\left(a_{i}^{*}\right) 1_{\mathrm{vac}} & =0
\end{aligned}
$$

where the action of $\lambda_{0}\left(a_{i}^{*}\right)$ on the monomials of degree $n \geq 1$ is determined inductively using the basic relations, is called the Fock representation.

It is easy to see that $\lambda_{0}\left(a_{i}\right)$ are the classical creation operators and $\lambda_{0}\left(a_{i}^{*}\right)$ are twisted annihilation ones. Evidently in this way we have constructed a representation of $W(T)$, but not yet a $*$-representation. To do it one has to supply the $\mathcal{T}(\mathcal{H})$ by the appropriate inner product (see [11]). This is where formula (4) comes in.

Definition 2. The Fock inner product (see [11]) is the unique semilinear Hermitian form $\langle,\rangle_{T}$ on $\mathcal{T}(\mathcal{H})$ such that

$$
\left\langle\lambda_{0}\left(a_{i}\right) X, Y\right\rangle_{T}=\left\langle X, \lambda_{0}\left(a_{i}^{*}\right) Y\right\rangle_{T}, \quad X, Y \in \mathcal{T}(\mathcal{H}) .
$$

Similarly to the definition of Fock representation, the Fock inner product on $\mathcal{T}(\mathcal{H})$ can be computed inductively. It is easy to see that for $X \in \mathcal{H}^{\otimes m}$, $Y \in \mathcal{H}^{\otimes n}, n \neq m$, we have $\langle X, Y\rangle_{T}=0$. On the components of powers 0,1 , the Fock inner product concides with the standard one. For any $X, Y \in$ $\mathcal{H}^{\otimes n}, n \geq 2$, we have

$$
\langle X, Y\rangle_{T}=\left\langle X, P_{n} Y\right\rangle,
$$

which agrees with (4) above. The operator $P_{n}=P_{n}(T)$ are given in (3).

Evidently, if we want to extend the Fock representation of $W(T)$ to the *-representation by Hilbert-space operators, we should require that all the operators $P_{n}, n=2, \ldots$, be positive semidefinite, and that the subspace

$$
\mathcal{I}=\bigoplus_{n \geq 2} \operatorname{ker} P_{n}
$$

determines the kernel of the Fock inner product. Consequently the Hilbertspace structure of the Fock space emerges.

\section{The structure of the Fock representation}

In this section we present some sufficient conditions posed on the operator $T$ for the positive-definite property of the Fock inner product, and we show that the kernel of the Fock representation is generated as a *-ideal by the kernel of the Fock inner product. In particular, when the Fock inner product is strictly positive definite (i.e., when it has zero kernel), the Fock representation $\pi_{F}$ is faithful, i.e., $\operatorname{ker}\left(\pi_{F}\right)=0$. 
There are several sufficient conditions on the operator $T$ for the Fock inner product to be positive. It was shown in [10] that for sufficiently small coefficients we have strict positivity of the Fock inner product. This result is a corollary of the stability of the universal enveloping $C^{*}$-algebra for the Wick algebra around the zero base point (see Sec. 4).

Theorem 1. If the operator $T$ satisfies the norm bound $\|T\|<\sqrt{2}-1$, then $P_{n}>0, n \geq 2$, where $>$ refers to strict positivity.

Another kind of sufficient condition is positivity of operator $T$ (see [11]).

Theorem 2. If $T \geq 0$ then $P_{n}>0, n \geq 2$.

In the present paper we will suppose that the operator $T$ satisfies the braid condition (6). It was shown by M. Bożejko and R. Speicher (see [12]) that, in this case, the operators $P_{n}, n \geq 2$, have a natural description in terms of quasimultiplicative operator-valued mappings on the Coxeter group $S_{n}$. The following is a corollary of a much more general result proved in [12] for mappings on the general Coxeter group.

Theorem 3. Let $T$ satisfy the braid condition (6) and suppose $-1 \leq T \leq$ 1. Then $P_{n} \geq 0$. Moreover, if $\|T\| \leq 1$, then $P_{n}>0$, and the operators of the Fock representation are bounded, i.e., the Fock representation is by bounded operators. (Recall, the Fock representation of the undeformed CCR-algebra is unbounded.)

We present a more precise version of this theorem. Namely, we give the description of kernel of $P_{n}$ in the degenerate case. As an immediate corollary of this result we have the strict positivity of $P_{n}, n \geq 2$, for braided $T$ satisfying the inequality $-1<T \leq 1$ (see [4]).

Theorem 4. Let $W(T)$ be a Wick algebra with braided operator $T$ satisfying the norm bound $\|T\| \leq 1$. Then for any $n \geq 1$,

$$
\operatorname{ker} P_{n+1}=\sum_{k+l=n-1} \mathcal{H}^{\otimes^{k}} \otimes \operatorname{ker}(1+T) \otimes \mathcal{H}^{\otimes^{l}}=\sum_{k=1}^{n} \operatorname{ker}\left(1+T_{k}\right) .
$$

Let us illustrate this result on the examples.

EXAMPLE 5. For $q_{i j}$-CCR we have the alternatives:

$-\left|q_{i j}\right|<1$ for any $i, j=1, \ldots, d$.

In this case $-1<T<1$ and the Fock inner product is strictly positive.

$-\left|q_{i j}\right|=1, i \neq j$.

For these values of parameters we have $-1 \leq T \leq 1$ and

$$
\operatorname{ker}(1+T)=\left\langle a_{j} a_{i}-q_{i j} a_{i} a_{j}, i<j\right\rangle .
$$


EXAmPle 6 . For the TCCR Wick algebra, we have $-1 \leq T \leq 1$, and

$$
\operatorname{ker}(1+T)=\left\langle a_{j} a_{i}-\mu a_{i} a_{j}, i<j\right\rangle .
$$

The following proposition shows that, for algebras with braided operator $T$, the kernel of the Fock representation is generated as a $*$-ideal by the kernel of the Fock inner product, i.e.,

$$
\mathcal{I}=\bigoplus_{n \geq 2} \operatorname{ker} P_{n}
$$

Proposition 2. Let $W(T)$ be a Wick algebra with braided operator $T$ and let the Fock representation $\lambda_{0}$ be positive (i.e., the Fock inner product is positive definite). Then

$$
\text { ker } \lambda_{0}=\mathcal{I} \otimes \mathcal{T}\left(\mathcal{H}^{*}\right)+\mathcal{T}(\mathcal{H}) \otimes \mathcal{I}^{*}
$$

Combining this proposition with Theorem 4, we get:

Theorem 5. Let $W(T)$ be a Wick algebra with the braided operator $T$, $-1 \leq T \leq 1$. Then the kernel of the Fock representation is generated as a *-ideal by $\operatorname{ker}(1+T)$.

This theorem implies that, for $q_{i j}$-CCR, $\left|q_{i j}\right|<1$, the Fock representation is faithful. For the TCCR Wick algebra, and for $q_{i j}$-CCR, the kernels of the Fock representations are generated by the families $a_{j} a_{i}-\mu a_{i} a_{j}, i<j$, and $a_{j} a_{i}-q_{i j} a_{i} a_{j}, i<j$, respectively; and hence the Fock representations of quotients of these algebras by the $*$-ideals generated by these families are faithful.

\section{Universal bounded representation}

In this section we discuss universal enveloping $C^{*}$-algebras for $q_{i j}$-CCR and Wick TCCR.

Let us recall that the universal $C^{*}$-algebra for a certain $*$-algebra $\mathcal{A}$ is also called the universal bounded representation. It is the $C^{*}$-algebra $\mathbf{A}$ with natural homomorphism $\psi: \mathcal{A} \rightarrow \mathbf{A}$ such that, for any homomorphism $\varphi: \mathcal{A} \rightarrow B$, where $B$ is a $C^{*}$-algebra, there exists a unique homomorphism $\theta: \mathbf{A} \rightarrow B$ satisfying $\theta \circ \psi=\varphi$. It can be obtained by the completion of $\mathcal{A} / J$ with the following $C^{*}$-seminorm on $\mathcal{A}$ :

$$
\|a\|=\sup _{\pi}\|\pi(a)\|
$$

where sup is taken over all bounded representations of $\mathcal{A}$, and $J$ is the kernel of this seminorm. Obviously this process requires that $\sup _{\pi}\|\pi(a)\|<\infty$ for any $a \in \mathcal{A}$. Note that for our examples this condition is satisfied.

The universal bounded representation for $q_{i j}$-CCR was studied in $[9,10]$. The following proposition follows from the main result of paper [10]. 
Proposition 3. Let $\mathbf{A}_{\left\{q_{i j}\right\}}$ be the universal enveloping $C^{*}$-algebra for $q_{i j}$ CCR, $\left|q_{i j}\right|<\sqrt{2}-1$. Then there exists the natural isomorphism

$$
\mathbf{A}_{\left\{q_{i j}\right\}} \cong \mathbf{A}_{0}
$$

where $\mathbf{A}_{0}$ is a $C^{*}$-algebra generated by the isometries $s_{i}, i=1, \ldots, d$, satisfying

$$
s_{i}^{*} s_{j}=0, i \neq j
$$

i.e., isomorphism with the Cuntz-Toeplitz algebra.

This implies that the Fock representation of $\mathbf{A}_{\left\{q_{i j}\right\}}$ is faithful.

Let us consider the $\mathbf{A}_{\left\{q_{i j}\right\}},\left|q_{i j}\right|=1$, for any $i \neq j$ and $q_{i i}:=q_{i},\left|q_{i}\right|<1$ (i.e., unimodular off-diagonal terms). In this case, we do not have stability on the whole set of parameters (see [18]).

Proposition 4. If for any $i \neq j$ we have $\left|q_{i j}\right|=1$, then $\mathbf{A}_{\left\{q_{i j}\right\}}$ is isomorphic to the $C^{*}$-algebra $\mathbf{A}_{0,\left\{q_{i}\right\}}$ generated by isometries $\left\{s_{i}, i=1, \ldots, d\right\}$ satisfying

$$
s_{i}^{*} s_{j}=q_{i j} s_{j} s_{i}^{*}, s_{j} s_{i}=q_{i j} s_{i} s_{j}, i \neq j,
$$

and the Fock representation of $\mathbf{A}_{\left\{q_{i j}\right\}}$ is faithful.

Finally for the universal $C^{*}$-algebra $\mathbf{A}_{\mu}$ for the Wick TCCR, we have the isomorphism $\mathbf{A}_{\mu} \cong \mathbf{A}_{0}$ for any $-1<\mu<1$, where the $C^{*}$-algebra $\mathbf{A}_{0}$ is generated by the partial isometries $\left\{s_{i}, i=1, \ldots, d\right\}$ satisfying the relations

$$
s_{i}^{*} s_{j}=\delta_{i j}\left(1-\sum_{k<i} s_{k} s_{k}^{*}\right), \quad i, j=1, \ldots, d .
$$

The Fock representation of $\mathbf{A}_{\mu}$ is faithful also (see [19]).

ACKnowledgements. P. J. was partially supported by the NSF under grants DMS-9700130 and INT-9722779. 


\section{References}

1. L.C. Biedenharn, The quantum group $\mathrm{SU}_{q}(2)$ and a q-analogue of the boson operators, J. Phys. A 22 (1989), L873-L878.

2. I.M. Burban and A.U. Klimyk, On spectral properties of q-oscillator operators, Lett. Math. Phys. 29 (1993), 13-18.

3. D.I. Fivel, Interpolation between Fermi and Bose statistics using generalized commutators, Phys. Rev. Lett. 65 (1990), 3361-3364.

4. P.E.T. Jørgensen, D.P. Proskurin and Yu. S. Samollenko, The kernel of Fock representations of Wick algebras with braided operator of coefficients, accepted for publication in Pacific J. Math., math-ph/0001011.

5. A.J. Macfarlane, On q-analogues of the quantum harmonic oscillator and the quantum group $\mathrm{SU}(2)_{q}$, J. Phys. A 22 (1989), 4581-4588.

6. W. Pusz and S.L. Woronowicz, Twisted second quantization, Rep. Math. Phys. 27 (1989), 251-263.

7. R.F. Werner, The free quon gas suffers Gibbs' paradox, Phys. Rev. D (3) 48 (1993), 2929-2934.

8. M. Bożejko and R. Speicher, An example of a generalized Brownian motion, Commun. Math. Phys. 137 (1991), 519-531.

9. K. Dykema and A. Nica, On the Fock representation of the q-commutation relations, J. Reine Angew. Math. 440 (1993), 201-212.

10. P.E.T. Jørgensen, L.M. Schmitt and R.F. Werner, q-canonical commutation relations and stability of the Cuntz algebra, Pacific J. Math. 165 (1994), 131-151.

11. Positive representations of general commutation relations allowing Wick ordering, J. Funct. Anal. 134 (1995), 33-99.

12. M. Bożejko and R. Speicher, Completely positive maps on Coxeter groups, deformed commutation relations, and operator spaces, Math. Ann. 300 (1994), 97-120.

13. W. Marcinek and R. Ralowski, On Wick algebras with braid relations, J. Math. Phys. 36 (1995), 2803-2820.

14. O.W. Greenberg, Particles with small violations of Fermi or Bose statistics, Phys. Rev. D (3) 43 (1991), 4111-4129.

15. W. Marcinek, On commutation relations for quons, Rep. Math. Phys. 41 (1998), 155-172.

16. P.E.T. Jørgensen and R.F. Werner, Coherent states of the q-canonical commutation relations, Commun. Math. Phys. 164 (1994), 455-471.

17. J. Cuntz, Simple $C^{*}$-algebras generated by isometries, Commun. Math. Phys. 57 (1977), 173-185.

18. D. Proskurin, Stability of a special class of $q_{i j}-C C R$ and extensions of higherdimensional noncommutative tori, to appear in Lett. Math. Phys.

19. D. Proskurin and Yu. Samoilenko, Stability of a $C^{*}$ algebra associated with the TCCR, submitted to Algebras and Representation Theory.

20. V. Ostrovskyı̆ and Yu. Samollenko, Introduction to the Theory of Representations of Finitely Presented *-Algebras, I: Representations by bounded operators, The Gordon and Breach Publishing Group, London, 1999.

21. D. P. Proskurin, Homogeneous ideals in Wick *-algebras, Proc. Amer. Math. Soc. 126 (1998), 3371-3376. 